\title{
Primary Selection for Uniform Display Response
}

\author{
Carlos E. Rodríguez-Pardo ${ }^{1}$ and Gaurav Sharma ${ }^{1}$ \\ Xiao-Fan Feng ${ }^{2}$ \\ ${ }^{1}$ ECE Dept. Univ. of Rochester, Rochester, NY, USA \\ ${ }^{2}$ Sharp Laboratories of America
}

\begin{abstract}
Traditional methodologies for primary selection usually consider the optimization of parameters that characterize the global performance of the display system, such as the luminance of the white point, gamut volume, and power consumption. We propose a methodology for primary design that optimizes a figure of merit designed to favor gamuts for which maximum luminance at each chromaticity is uniformly related to the corresponding maximum luminance over the set of optimal colors. We contrast the results obtained with the proposed methodology with those obtained by an alternative strategy based on the optimization of gamut volume, and analyze differences in performance between these approaches for both three and four primary systems. Results indicate that the global vs local design choices result in significantly different primary designs.
\end{abstract}

Keywords: primary selection, display design, optimal colors, maximum luminance, display gamut

\section{INTRODUCTION}

The current diversity and flexibility of color display technologies offers the possibility to address different display requirements through the selection of suitable primaries. The availability of narrowband sources, like LEDs, and color filters with a sharp cut-off, such as the ones used for LCDs, makes possible primary sets that widen display gamuts. The use of narrowband primaries, however, also incurs two main disadvantages: observer metamerism, ${ }^{1}$ and potential loss of efficiency with narrowband filters. As an alternative, the use of more than the traditional three primaries also allows expansion of the gamut, ${ }^{2,3}$ and brings benefits for power consumption, ${ }^{4,5}$ viewing angle, ${ }^{6}$ among others.

Regardless of the technology, a common practice in the process of design is the optimization of certain figures of merit, that include, among others, white luminance, gamut volume (or gamut area) in a perceptual space, and coverage of standard color data sets. ${ }^{7-11}$ These metrics*, in general, characterize globally the gamut of the display, but may overlook the actual performance in local regions of the gamut. The maximum luminance for certain chromaticities, for example, may vary significantly depending on the particular choice of primaries, selected from sets having the same gamut volume and white luminance. Although there isn't a unique way of specifying what constitutes a good chromaticity reproduction, in this paper we focus in exploring metrics that quantify the local display performance, and propose strategies for primary design intended to produce gamuts whose maximum luminance gain is proportionally uniform over the gamut of mathematically feasible surface colors, the latter being characterized by the optimal surface colors. ${ }^{12}$

Some prior work has already explored this direction. Hinnen et. al. ${ }^{13}$ proposed a methodology for qualitative comparison between display gamuts based on the definition of an index that compares luminance for each chromaticity in the display gamut with the luminance of optimal colors. Wen ${ }^{14}$ presented a primary selection method by computing for pairs of hue and luminance, the ratio between the maximum chroma in the gamut, and the corresponding maximum chroma in set of optimal colors, although with different motivation.

Further author information:

C.R.: E-mail: pardo@ece.rochester.edu, Telephone: 15852758122

This work is supported in part by a grant from Sharp Labs America.

${ }^{*}$ We use the term metric generically, recognizing that several of the figures of merit are not truly metrics in a mathematically formal sense.

Color Imaging XIX: Displaying, Processing, Hardcopy, and Applications, edited by Reiner Eschbach, Gabriel G. Marcu, Alessandro Rizzi, Proc. of SPIE-IS\&T Electronic Imaging, SPIE Vol. 9015, 90150I · @ 2014 SPIE-IS\&T · CCC code: 0277-786X/14/\$18 · doi: 10.1117/12.2041157 
As part of ongoing work on exploring display design strategies, in this manuscript we propose a metric to describe local performance in chromaticity space, present designs based on the optimization of this metric and contrast them with designs obtained with alternative methods. ${ }^{7}$

The rest of the document is organized as follows. Section 2 introduces the display metric, based on which, we define the display primary design optimization problem in Section 3. Discussion of the results are presented in Section 4, and the conclusions are presented in Section 5.

\section{DISPLAY LUMINANCE}

\subsection{Display gamut}

The set of colors that a device is able to display is known as the gamut ${ }^{\dagger}$. For a system with $K$ primaries with $C I E X Y Z$ tristimulus values $\mathbf{p}_{1}, \mathbf{p}_{2}, \cdots, \mathbf{p}_{K}$, the gamut in the $C I E X Y Z$ space, denoted by $\mathcal{G}_{\mathbf{P}}^{X Y Z}$, where $\mathbf{P}$ is the matrix of primaries, $\mathbf{P}=\left[\mathbf{p}_{1}, \mathbf{p}_{2}, \cdots, \mathbf{p}_{K}\right]$, is defined as the set of primary combinations described by,

$$
\mathcal{G}_{\mathbf{P}}^{X Y Z}=\left\{\mathbf{P} \boldsymbol{\alpha} \mid \boldsymbol{\alpha} \in[0,1]^{K}\right\}
$$

where $\boldsymbol{\alpha}=\left[\alpha_{1}, \alpha_{2}, \cdots, \alpha_{K}\right]$ is the vector of relative amplitudes, taking on values between 0 and 1 for each component. Gamut representations can be obtained for other color spaces from the definition presented in (1), by applying the corresponding color transformation. ${ }^{15}$ In this paper, we represent each of the colors with the triad $\left(Y, u^{\prime}, v^{\prime}\right)$, formed by the $C I E 1976$ chromaticity coordinates $u^{\prime}, v^{\prime}$ and the $Y$ component of the CIEXYZ tristimulus. For simplicity in notation, $\mathcal{G}_{\mathbf{P}}$ is used as a generic term to describe the gamut of the display in any color space.

\subsection{Relative luminance distribution}

Methodologies for design usually focus on two important luminance metrics: The luminance at the white point, which is the maximum luminance of the gamut, and the minimum luminance of the primaries. Once both quantities are fixed, the maximum luminance that the display is able to reproduce for each chromaticity will lie between these two values. However, the actual values vary depending on the primary selection. For a given display with matrix of primaries $\mathbf{P}$, it is possible to obtain an idea of the relative distribution of luminance for each chromaticity $\left(u^{\prime}, v^{\prime}\right)$ by computing the relative index, $\zeta\left(u^{\prime}, v^{\prime}\right)$, defined as,

$$
\zeta_{\mathbf{P}}\left(u^{\prime}, v^{\prime}\right)=\frac{Y_{\max }^{\mathcal{G}_{\mathbf{P}}}\left(u^{\prime}, v^{\prime}\right)}{Y_{W P}}
$$

where,

$$
Y_{\text {max }}^{\mathcal{G}_{\mathbf{P}}}(u, v)= \begin{cases}\max \left\{Y \mid\left(Y, u^{\prime}, v^{\prime}\right) \in \mathcal{G}_{\mathbf{P}}\right\}, & \text { if }\left(u^{\prime}, v^{\prime}\right) \in \mathcal{G}_{\mathbf{P}} \\ 0, & \text { otherwise }\end{cases}
$$

which is essentially the maximum luminance that the display can reproduce for a chromaticity $\left(u^{\prime}, v^{\prime}\right)$. Note that $Y_{\max }^{\mathcal{G}_{\mathrm{P}}}$ has been defined for all chromaticity points, which is exploited later in the paper. Figure 1(a) shows the relative luminance distribution for a set of primaries matching the REC709 standard specifications with white point at D65. As expected, lower values of luminance are found at the primaries, and the luminance increases toward the white point. The graph can be used for a qualitative evaluation of the gamut, where it becomes evident how certain chromaticities regions are favored in luminance by the choice of certain primaries. This function alone, however, may not necessarily express how well the gamut "covers" a given chromaticity, since a coverage criterion has not been defined yet. A more concrete evaluation can be obtained if $\zeta_{\mathbf{P}}\left(u^{\prime}, v^{\prime}\right)$ is compared to a luminance reference, like the set of optimal colors, ${ }^{12}$ for which the gamut is denoted by $\mathcal{G}_{\text {opt }}$, and whose luminance distribution, $\zeta_{\text {opt }}\left(u^{\prime}, v^{\prime}\right)$, is shown in Fig. 1(b).

\footnotetext{
${ }^{\dagger}$ We adopt a notational convention identical to our prior work. ${ }^{7}$
} 


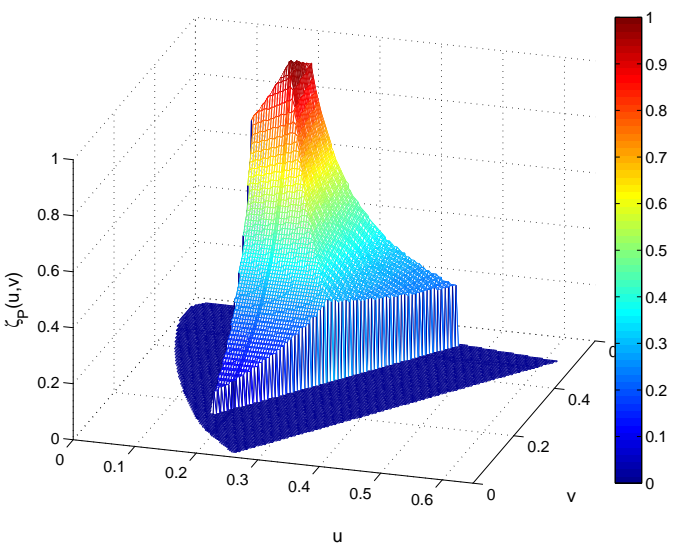

(a) REC709

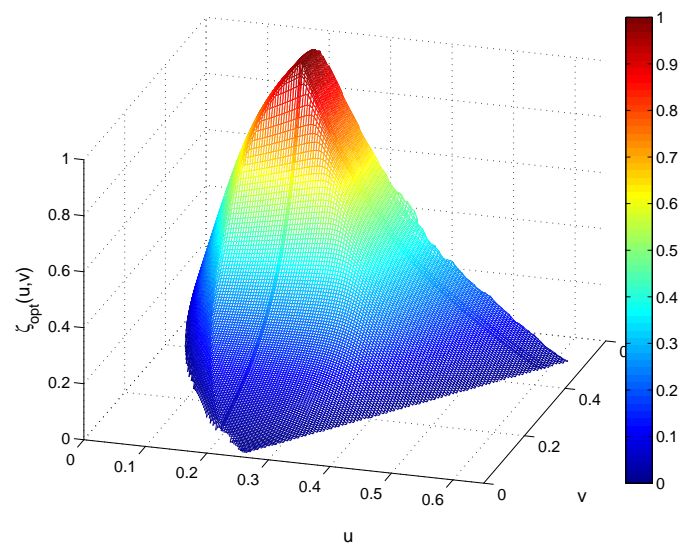

(b) Optimal colors

Figure 1. Relative luminance distribution function $\zeta\left(u^{\prime}, v^{\prime}\right)$, for the REC709 standard, and the set of optimal colors. Both under D65 illuminant/white point

\subsection{Relative luminance deviation}

The set of optimal colors constitutes a natural reference for comparison. For a given chromaticity, the optimal color has the maximum luminance that can be found by a material colored with that chromaticity, under a given illuminant. ${ }^{12,16}$ In typical usage settings, one is interested in displaying surface colors, as rendered under an illuminant, on the display. Therefore, the luminance of optimal colors can be used as an indicator of how well a chromaticity is reproduced within the display color gamut. A good chromaticity reproduction is obtained if the device is able to reproduce colors of all chromaticity with relative luminance levels close to the optimal ones.

The difference between the relative luminance distributions of the display and the set of optimal colors, sharing the same white point reference, is called here as the relative luminance deviation, denoted by $\delta_{\mathbf{P}}\left(u^{\prime}, v^{\prime}\right)$, and computed as,

$$
\delta_{\mathbf{P}}\left(u^{\prime}, v^{\prime}\right)=\left|\frac{\zeta_{\mathbf{P}}\left(u^{\prime}, v^{\prime}\right)-\zeta_{\text {opt }}\left(u^{\prime}, v^{\prime}\right)}{\zeta_{\text {opt }}\left(u^{\prime}, v^{\prime}\right)}\right| .
$$

The function defined in (4) extends an index proposed earlier. ${ }^{13}$ The are two differences, subtle but important, that allow us to formulate a figure of merit for optimization based on this quantity. First, by using $\zeta$ instead of the regular $Y$ luminance values, the function is defined for all points inside the gamut of optimal colors. This offers a device independent definition, which allows consistent evaluation across different gamuts, and facilitates comparisons between primary designs, an important and useful attribute for a criterion for optimization. Second, $\delta_{\mathbf{P}}\left(u^{\prime}, v^{\prime}\right)$ has been defined as a magnitude. By using the absolute value, we consider deviations in both directions: reproductions of color with luminance lower, and higher, than the luminance of optimal colors, both of which are penalized. A lower luminance is undesirable because it represents chromaticities that cannot be reproduced with adequate luminance. A higher luminance is not useful since those colors are not found in objects. Values of zero for $\delta$ are desirable and will represent a balance between being able to reproduce a color, and using the lowest possible optical power. Another subtle difference relies on the fact that here the computation is made only for set of primaries that share the same white point with point as the set of optimal colors.

The deviation function $\delta_{\mathbf{P}}\left(u^{\prime}, v^{\prime}\right)$ can be used as metric of display performance for the chromaticity $\left(u^{\prime}, v^{\prime}\right)$. Figure 2 shows the deviation curve for a set of REC709 primaries. According to the graph, this configuration exhibits clear differences in performance for different chromaticities. For example, the regions around the blue primary attain low values of $\delta_{\mathbf{P}}$, implying a reproduction of maximum luminance close to the reference, despite the low levels of relative luminance that can be seen in Fig.1(a). On the other hand, chromaticities close to red, specially the yellow region, are sub-optimally reproduced, as compared to other regions in the gamut. This statement can be verified by checking Figures 2(b) and 2(c), which show the projection along the $v^{\prime}$ and $u^{\prime}$ axis, respectively, of the relative luminance distribution for the display, $\zeta_{\mathbf{P}}$, and for the reference, $\zeta_{o p t}$. Note in 
Fig. 2(b) that the maximum display luminance for chromaticities between the white point and the red primary is significantly lower than the luminance of optimal colors.

To quantify overall display performance, we average the values of $\delta_{\mathbf{P}}\left(u^{\prime}, v^{\prime}\right)$ over the optimal color gamut. Specifically, we create a grid on the chromaticity space of $N$ pairs of chromaticities, $\left\{\left(u_{n}^{\prime}, v_{n}^{\prime}\right) \in \mathcal{G}_{\text {opt }}, 1 \leq n \leq N\right\}$, that cover the optimal color gamut. We use the grid to evaluate the display mean deviation, $\Delta_{\mathbf{P}}$, as,

$$
\Delta_{\mathbf{P}}=\frac{1}{N} \sum_{1 \leq n \leq N} \delta_{\mathbf{P}}\left(u_{n}^{\prime}, v_{n}^{\prime}\right)
$$

and we propose it as an index of uniformity. A display is uniform if it is able to reproduce all the chromaticities inside its gamut with the same luminance, relative to the optimal set of colors. The ideal uniform device has a $\Delta_{\mathcal{G}_{\mathrm{P}}}=0$ and therefore, a gamut matching the gamut of optimal colors. Deviations from this target, corresponding either to higher or lower luminance, or to chromaticities that are outside of the device gamut, are both penalized.

The term $\frac{1}{N}$ in Equation (5) assumes that all chromaticities inside the optimal colors are equally important, which is the assumption used in this paper. For a more accurate computation, the term might be changed based other criteria, like the specific interest in certain gamut regions, or the statistical distribution of surface colors.

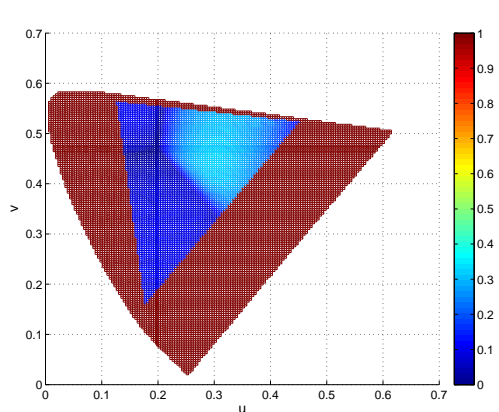

(a) $\delta_{\mathbf{P}}\left(u^{\prime}, v^{\prime}\right)$

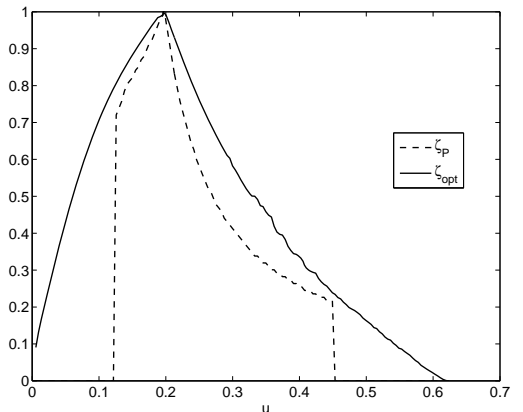

(b) $\zeta_{\mathbf{P}}\left(u^{\prime}, \cdot\right)$

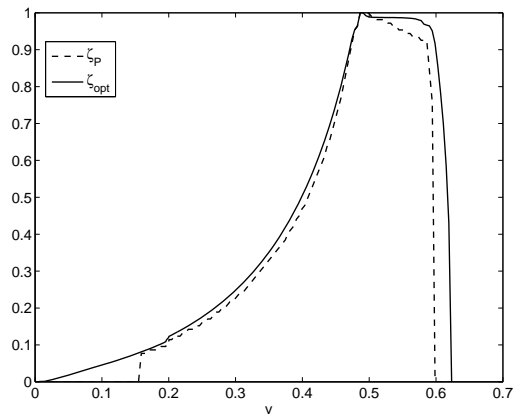

(c) $\zeta_{\mathbf{P}}\left(\cdot, v^{\prime}\right)$

Figure 2. Relative luminance functions for a gamut with REC709 primaries and D65 white point: The luminance deviation $\delta_{\mathbf{P}}\left(u^{\prime}, v^{\prime}\right)$, and the luminance distributions $\zeta_{\mathbf{P}}\left(u^{\prime}, \cdot\right), \zeta_{\mathbf{P}}\left(\cdot, v^{\prime}\right)$, as the projections of $\zeta_{\mathbf{P}}\left(u^{\prime}, v^{\prime}\right)$ along the $v^{\prime}$ and $u^{\prime}$ axis, respectively.

\section{METHODOLOGY FOR OPTIMAL PRIMARY SELECTION FOR UNIFORM DISPLAYS}

\subsection{Spectral modeling and constraint considerations for a display system}

We present a methodology for primary design based on the optimization of $\Delta_{\mathbf{P}}$ in the spectral domain, following a methodology similar to the one proposed in our earlier work, ${ }^{7}$ though with the updated objective function proposed here. The spectral characterization of the primaries allows designs that closely follow technological requirements. In order to also allow a generic use of the methodology, we approximate the spectral distribution of the primaries as normalized Gaussian functions, and use the mean, amplitude, and variance, as parameters for design. For a system with $K$ primaries, the spectra for the primary $\mathbf{p}_{i}, i, 1 \leq i \leq K$ is denoted $p_{i}(\lambda)$ and is defined by the Gaussian function with parameters $a_{i}, \lambda_{i}, \sigma_{i}$ as,

$$
p_{i}(\lambda)=a_{i} \frac{1}{\sqrt{2 \pi \sigma_{i}^{2}}} \exp \left\{-\frac{\left(\lambda-\lambda_{i}\right)^{2}}{2 \sigma_{i}^{2}}\right\} .
$$

With this definition, an important properties like the optical power Power $_{i}$ can be easily be computed as,

$$
\text { Power }_{i}=\int p_{i}(\lambda) d(\lambda)=a_{i}
$$




\subsubsection{Constraints on design paramters}

Optical Power: As a common restriction, displays operate under a certain electric power limit, which correlates with the optical power of the system. According to the display model introduce in Section 3.1, the total optical power of the system, $\mathcal{P}$, is computed as the sum of optical power of the primaries,

$$
\mathcal{P}=\sum_{i=1}^{K} a_{i} .
$$

White Balance: The brightest color in the display, or white point, is denoted as $\mathbf{w}_{\mathbf{P}}$, and can be computed as the sum of all primaries. To ensure that the white point is located at a chromaticity pair of a given reference, denoted as $\mathbf{w}_{r}$, the relative amplitudes of the primaries is modified. In this problem, we are interested in search for designs for which primaries are already balanced. For three primaries, the white balance can be expressed as set of three linear equations and three variables that have unique solution, provided a set of linearly independent primaries. For multiprimary systems, the solution is not unique, and additional requirements, such as maximizing white luminance, are defined to constraint the solutions. ${ }^{17}$

\subsection{Optimal design formulation}

For a given illuminant with white point $\mathbf{w}_{r}$, and a grid of chromaticities $\left\{\left(u_{n}^{\prime}, v_{n}^{\prime}\right) \in \mathcal{G}_{\text {opt }}, 1 \leq n \leq N\right\}$, the spectral parameters for the primaries of a uniform $K$ - primary display are found by solving the following optimization problem,

$$
\begin{aligned}
& \min _{\mathbf{a}, \lambda, \sigma} \Delta_{\mathbf{P}_{\mathbf{a}, \lambda, \sigma}} \\
& \text { subject to: } \mathbf{w}_{\mathbf{P}}=\mathbf{w}_{r}, \\
& \sum_{i=1}^{K} a_{i}=\mathcal{P}_{\max }, \\
& 0 \leq \mathbf{a}, \\
& 380 \mathrm{~nm} \leq \lambda_{i} \leq 680 \mathrm{~nm}, i=1,2, . . K, \\
& \sigma_{\min } \leq \sigma_{i} \leq \sigma_{\max }, i=1,2, . . K,
\end{aligned}
$$

where $\mathcal{P}_{\max }, \sigma_{\min }, \sigma_{\max }$, are parameters that can change according to the characteristics of the technology.

The problem is not convex in general, which requires the use of a global optimization method to obtain meaningful results. We use differential evolution, ${ }^{18,19}$ which is a broadly applicable global optimization technique.

\section{RESULTS AND DISCUSSION}

\subsection{Three primary designs}

In our first experiment we apply the formulation in (9) for a three primary display, and allow a wide range for the bandwidth of the primaries, by setting $\sigma_{\min }=1 \mathrm{~nm}, \sigma_{\max }=100 \mathrm{~nm}$, and unitary power, $\mathcal{P}_{\max }=1$. The parameters of the configuration obtained are described in Table 3. The optimal deviation for this design can be seen in Fig.3(a), where it can be appreciated how saturated the primaries are (note the $\sigma$ values are close to the lower bound), and how they are distributed along the spectral locus. It can also be appreciated that most of the chromaticities have a $\delta_{\mathbf{P}}$ value zero or very close to zero, but there are others, specially around the primaries, with very high values. These high deviations are due to the reproduction of chromaticities with luminance higher than the corresponding luminance attained by the optimal colors, which can be corroborated by checking Fig. $3(\mathrm{~b})$, that shows how $\zeta_{\mathbf{P}}$ is significantly greater than $\zeta_{\mathbf{P}}$ in regions around the red and green primaries. This high deviation for some regions of saturated colors is compensated by the fact that the display is able to reproduce a significant portion of the gamut of optimal colors. 
Saturated primaries are expected, since, by definition, $\Delta_{\mathbf{P}}$ favors primary sets whose gamut is similar to the gamut of the optimal set of colors, and therefore, in the limiting case when very narrowband primaries are permitted, minimizing $\Delta_{\mathbf{P}}$ can be interpreted as an indirect way of maximizing chromaticity coverage of set of the optimal colors. However, saturated primaries implies very high values of $\delta$, and therefore primaries for the optimal uniform are not completely saturated and thus, do not coincide with the primaries designed to maximize the coverage of surface colors.

As a next experiment, we contrast the properties of the primaries obtained by this methodology, with the primaries that maximize the gamut volume in a perceptual space $C I E L U V$, denoted $V_{\mathbf{P}}^{L U V}$, following a methodology presented previously. ${ }^{7}$ The parameters obtained from this methodology under identical constraints are listed in Table 1, and the corresponding deviation and distributions curves are shown in Fig. 4. It is clear that the configuration covers more chromaticities, as expected, with a very saturated set of primaries. Figure 4(b) shows the high luminance of the primaries, and how the configuration has very high values of $\delta_{\mathbf{P}}$ in a broader region around the primaries, and therefore, a higher $\Delta_{\mathbf{P}}$.

Finally, we compute the optimal primaries when the bandwidth of the display primary spectra is not allowed to be very narrow, by setting $\sigma_{\min }=50$. We obtain a set of primaries whose deviation and distributions curves are shown in Fig. 5. In this case the primaries are a balance between the reproduction of more chromaticities and uniform luminance gain over the chromaticities. It is important to highlight that the penalty for not reproducing a chromaticity is uniformly 1 across the chromaticity space, while the penalty for differences in luminance depends on the corresponding relative differences. Since the luminance differences are not high for this scenario, the optimal primaries tend to be as saturated as possible, providing for broader coverage.

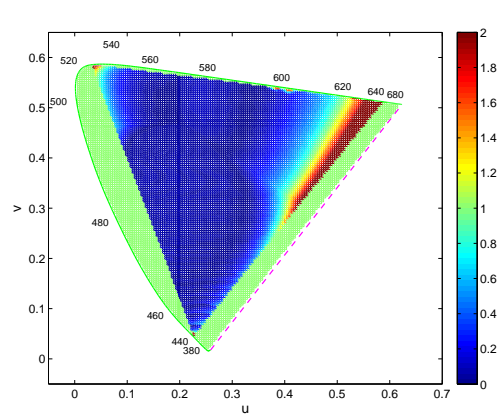

(a) $\delta_{\mathbf{P}}\left(u^{\prime}, v^{\prime}\right)$

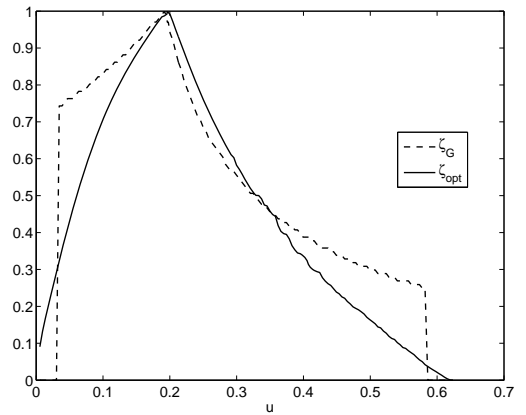

(b) $\zeta_{\mathbf{P}}\left(u^{\prime}, \cdot\right)$

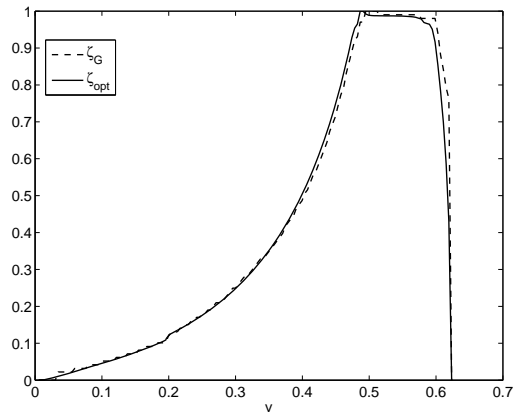

(c) $\zeta_{\mathbf{P}}\left(\cdot, v^{\prime}\right)$

Figure 3. Relative luminance functions for the optimal configuration for a three primary system with minimum $\Delta_{\mathbf{P}}$, when $\sigma_{\min }=1 \mathrm{~nm}$ : The luminance deviation $\delta_{\mathbf{P}}\left(u^{\prime}, v^{\prime}\right)$, and the luminance distributions $\zeta_{\mathbf{P}}\left(u^{\prime}, \cdot\right), \zeta_{\mathbf{P}}\left(\cdot, v^{\prime}\right)$, as the projections of $\zeta_{\mathbf{P}}\left(u^{\prime}, v^{\prime}\right)$ along the $v^{\prime}$ and $u^{\prime}$ axis, respectively.

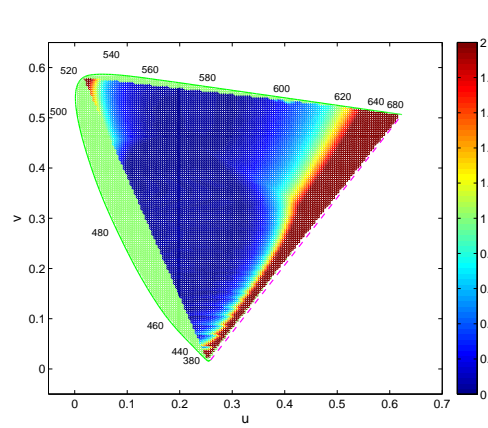

(a) $\delta_{\mathbf{P}}\left(u^{\prime}, v^{\prime}\right)$

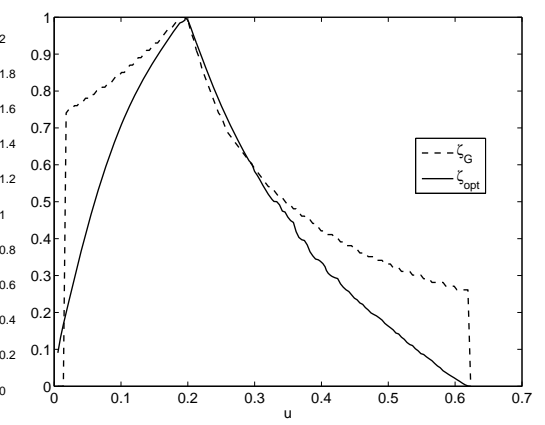

(b) $\zeta_{\mathbf{P}}\left(u^{\prime}, \cdot\right)$

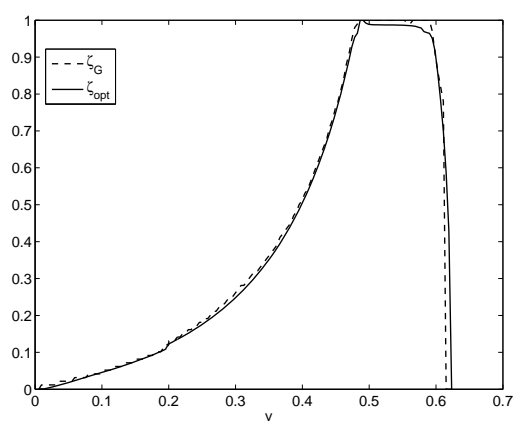

(c) $\zeta_{\mathbf{P}}\left(\cdot, v^{\prime}\right)$

Figure 4. Relative luminance functions for the optimal configuration for a three primary system with maximum $V_{\mathbf{P}}^{L U V}$, when $\sigma_{m i n}=1 n m$ : The luminance deviation $\delta_{\mathbf{P}}\left(u^{\prime}, v^{\prime}\right)$, and the luminance distributions $\zeta_{\mathbf{P}}\left(u^{\prime}, \cdot\right), \zeta_{\mathbf{P}}\left(\cdot, v^{\prime}\right)$, as the projections of $\zeta_{\mathbf{P}}\left(u^{\prime}, v^{\prime}\right)$ along the $v^{\prime}$ and $u^{\prime}$ axis, respectively. 


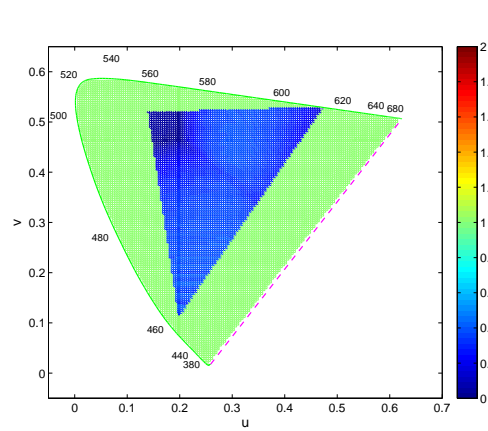

(a) $\delta_{\mathbf{P}}\left(u^{\prime}, v^{\prime}\right)$

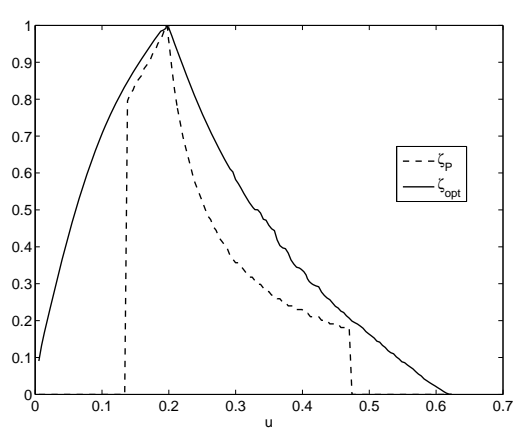

(b) $\zeta_{\mathbf{P}}\left(u^{\prime}, \cdot\right)$

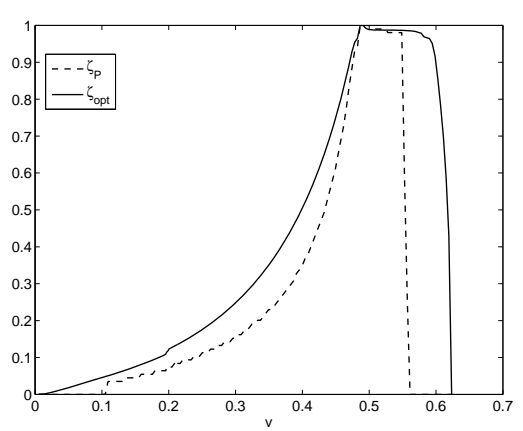

(c) $\zeta_{\mathbf{P}}\left(\cdot, v^{\prime}\right)$

Figure 5. Relative luminance functions for the optimal configuration for a three primary system with minimum $\Delta_{\mathbf{P}}$, when $\sigma_{\min }=50 \mathrm{~nm}$ : The luminance deviation $\delta_{\mathbf{P}}\left(u^{\prime}, v^{\prime}\right)$, and the luminance distributions $\zeta_{\mathbf{P}}\left(u^{\prime}, \cdot\right), \zeta_{\mathbf{P}}\left(\cdot, v^{\prime}\right)$, as the projections of $\zeta_{\mathbf{P}}\left(u^{\prime}, v^{\prime}\right)$ along the $v^{\prime}$ and $u^{\prime}$ axis, respectively.

\begin{tabular}{l|l|ccc|ccc|ccc}
\hline Design criterion & & $\lambda$ & $\sigma$ & $a$ & $u^{\prime}$ & $v^{\prime}$ & $Y$ & $Y_{W P}$ & $\Delta_{\mathbf{P}}$ & $V_{\mathbf{P}}^{L U V}$ \\
\hline & $\mathbf{p}_{1}$ & 444.68 & 5.83 & 0.0635 & 0.2253 & 0.0451 & 1.310 & & & \\
$\min \Delta_{\mathbf{P}}$ & $\mathbf{p}_{2}$ & 522.97 & 2.13 & 0.1089 & 0.0310 & 0.5853 & 56.520 & 76.84 & 0.4836 & $2.7576 \mathrm{e} 6$ \\
$\left(\sigma_{\min }=1 n m\right)$ & $\mathbf{p}_{3}$ & 686.25 & 25.91 & 0.8277 & 0.5867 & 0.5120 & 19.019 & & & \\
\hline & $\mathbf{p}_{1}$ & 420.75 & 4.08 & 0.0244 & 0.2508 & 0.0181 & 0.081 & & & \\
$\max V_{\mathbf{P}}^{L U V}$ & $\mathbf{p}_{2}$ & 516.44 & 1.03 & 0.0207 & 0.0153 & 0.5794 & 9.012 & 12.20 & 1.5421 & $3.1131 \mathrm{e} 6$ \\
$\left(\sigma_{\min }=1 n m\right)$ & $\mathbf{p}_{3}$ & 698.39 & 4.21 & 0.9549 & 0.6232 & 0.5065 & 3.107 & & & \\
\hline & $\mathbf{p}_{1}$ & 382.38 & 50.25 & 0.3174 & 0.1964 & 0.1099 & 3.491 & & & \\
$\min \Delta_{\mathbf{P}}$ & $\mathbf{p}_{2}$ & 525.48 & 50.03 & 0.2368 & 0.1377 & 0.5192 & 92.228 & 116.13 & 0.7406 & $9.3995 \mathrm{e} 5$ \\
$\left(\sigma_{\min }=50 \mathrm{~nm}\right)$ & $\mathbf{p}_{3}$ & 699.75 & 50.03 & 0.4457 & 0.4732 & 0.5288 & 20.413 & & & \\
\hline
\end{tabular}

Table 1. Optimal designs for three primary displays.

\subsection{Four Primary Designs}

We also used the formulation in (9) for a four primary display, with $\sigma_{\min }=1 \mathrm{~nm}, \sigma_{\max }=100 \mathrm{~nm}$, and $\mathcal{P}_{\max }=1$. The results are shown in Fig. 6 . The optimal design offers three saturated primaries associated with the perception of red, green, blue, and a fourth primary located between the yellow and red regions, mostly associated with the perception of orange. Figure 6(b) shows the relative luminance distributions for the reference and the display, and offers an explanation for the role of the extra primary in minimizing $\Delta_{\mathbf{P}}$. The relative luminance of the red primary is reduced considerably when compared with the configurations computed for the three primary scenario. From the graph, one can appreciate the contribution of the extra primary. The presence of the additional primary allows the reduction of excess luminance of the red primary, and therefore, a significant reduction of $\delta_{\mathbf{P}}$. The action of the primary helps not only saturated colors, but colors inside the gamut also improve their $\delta_{\mathbf{P}}$ value, since the relative luminance of green is reduced as well. It can be seen that the uniformity of the reproduction across the gamut is significantly improved.

We also obtain a configuration that maximizes the gamut volume $V_{\mathbf{P}}^{L U V}$. The primaries are more saturated, and the fourth primary is located around green, increasing the luminance gain for chromaticities in that region, and generating a higher score for $\Delta_{\mathbf{P}}$.

\section{CONCLUSIONS}

We have presented a framework for the evaluation of the local performance of display systems based on an evaluation of display response over the set of optimal colors. We extended previous work, ${ }^{13}$ by defining the relative luminance distribution, $\zeta_{\mathbf{P}}$, and the relative luminance deviation, $\delta_{\mathbf{P}}$, and use these as metrics for evaluation of the local performance. The function $\delta_{\mathbf{P}}$, in particular, captures relevant information about the reproduction of each chromaticity, like coverage and efficiency, which have effects on the overall performance of the display. We evaluate this effect, by defining the mean deviation, $\Delta_{\mathbf{P}}$, and proposing it as a figure of merit for display design. We develop a methodology for primary selection that minimizes this figure of merit. 


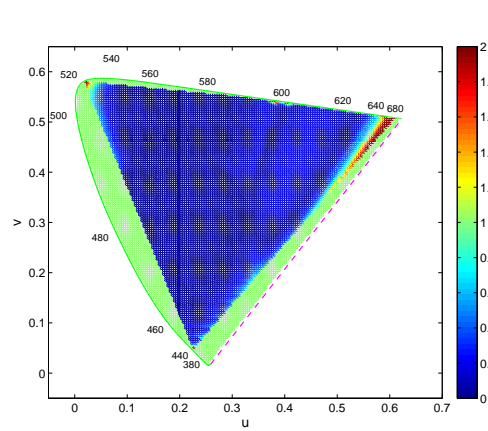

(a) $\delta_{\mathbf{P}}\left(u^{\prime}, v^{\prime}\right)$

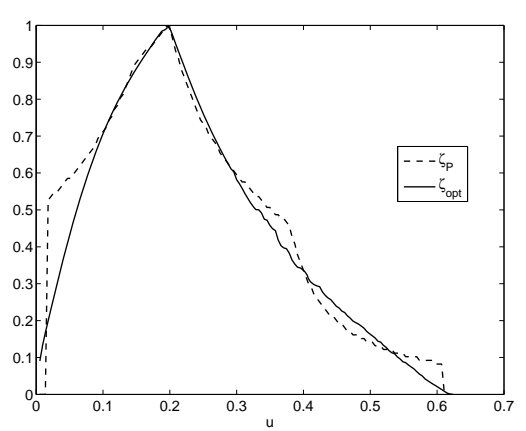

(b) $\zeta_{\mathbf{P}}\left(u^{\prime}, \cdot\right)$

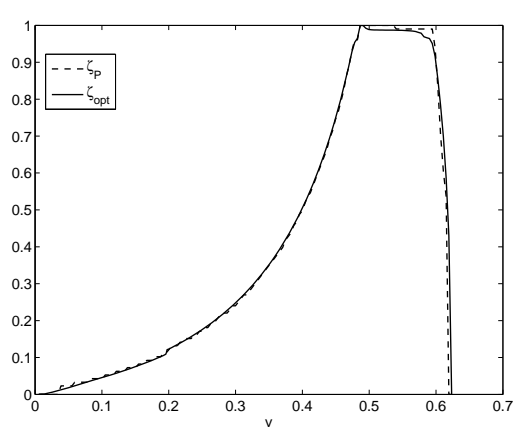

(c) $\zeta_{\mathbf{P}}\left(\cdot, v^{\prime}\right)$

Figure 6. Relative luminance functions for the optimal configuration for a four primary system with minimum $\Delta_{\mathbf{P}}$, when $\sigma_{m i n}=1 n m$ : The luminance deviation $\delta_{\mathbf{P}}\left(u^{\prime}, v^{\prime}\right)$, and the luminance distributions $\zeta_{\mathbf{P}}\left(u^{\prime}, \cdot\right), \zeta_{\mathbf{P}}\left(\cdot, v^{\prime}\right)$, as the projections of $\zeta_{\mathbf{P}}\left(u^{\prime}, v^{\prime}\right)$ along the $v^{\prime}$ and $u^{\prime}$ axis, respectively.

\begin{tabular}{l|l|ccc|ccc|ccc}
\hline Design criterion & & $\lambda$ & $\sigma$ & $a$ & $u^{\prime}$ & $v^{\prime}$ & $Y$ & $Y_{W P}$ & $\Delta_{\mathbf{P}}$ & $V_{\mathbf{P}}^{L U V}$ \\
\hline \multirow{3}{*}{$\Delta_{\mathbf{P}}$} & $\mathbf{p}_{1}$ & 443.23 & 10.12 & 0.1391 & 0.2239 & 0.0470 & 2.829 & & & \\
$\left(\sigma_{\min }=1 n m\right)$ & $\mathbf{p}_{2}$ & 516.79 & 1.83 & 0.1862 & 0.0163 & 0.5798 & 82.084 & 156.51 & 0.3110 & $2.5350 \mathrm{e} 6$ \\
& $\mathbf{p}_{3}$ & 627.67 & 51.83 & 0.2249 & 0.3250 & 0.5487 & 58.551 & & & \\
& $\mathbf{p}_{4}$ & 668.79 & 10.59 & 0.4498 & 0.6099 & 0.5085 & 13.049 & & & \\
\hline \multirow{2}{*}{$\max V_{\mathbf{P}}^{L U V}$} & $\mathbf{p}_{1}$ & 414.49 & 10.73 & 0.0952 & 0.2488 & 0.0204 & 0.252 & & & \\
$\left(\sigma_{\min }=1 n m\right)$ & $\mathbf{p}_{2}$ & 497.84 & 3.788 & 0.0478 & 0.6177 & 0.5073 & 9.738 & 41.91 & 0.9111 & $3.2646 \mathrm{e} 6$ \\
& $\mathbf{p}_{3}$ & 522.01 & 1.031 & 0.0410 & 0.0282 & 0.5850 & 20.861 & & & \\
& $\mathbf{p}_{4}$ & 679.54 & 8.281 & 0.8160 & 0.6177 & 0.5073 & 11.067 & & & \\
\hline \hline
\end{tabular}

Table 2. Optimal designs for four primary displays

In general the designs obtained by the proposed methodology show a better trade-off between optical power and color coverage, as compared with a display design methodology based on the optimization of a global parameter, like gamut volume. We also showed how minimizing $\Delta_{\mathbf{P}}$ has different interpretations according to the constraints imposed on the problem. When saturated primaries are allowed, the methodology favors the reduction of extra luminance, while if broad-band primaries are imposed, the methodology searches for configurations that expand the coverage of the set of optimal colors. In both scenarios, chromaticities inside the gamut tend to be reproduced with a maximum luminance that is uniformly related to the corresponding maximum luminance over the set of optimal colors.

We evaluate the methodology for three and four primary systems. For three primary systems, the methodology yields a set of saturated red, green and blue primaries. For the particular case of four primaries configurations allowing narrowband spectra, the optimal design offers three saturated primaries associated with the perception of red, green, blue, and a fourth primary located between the yellow and red regions, mostly associated with the perception of orange. We analyzed the effect and the benefits of the fourth primary in increasing luminance efficiency overall the gamut. It is interesting to highlight that the Quattron ${ }^{\mathrm{TM}},{ }^{20}$ a four primary commercial display, includes a yellow primary, although in this paper that particular configuration was not evaluated.

Finally, the approach adopted in this paper, by evaluating first local display properties and then defining a global figure of merit, is useful in the analysis of trade-offs, and can be applied for the evaluation of other display characteristics.

\section{REFERENCES}

[1] Ramanath, R., "Minimizing observer metamerism in display systems," Color Res. Appl. 34(5), 391-398 (2009).

[2] Teragawa, M., Yoshida, A., Yoshiyama, K., Nakagawa, S., Tomizawa, K., and Yoshida, Y., "Review paper: Multi-primary-color displays: The latest technologies and their benefits," Journal of the Society for Information Display 20(1), 1-11 (2012). 
[3] Cheng, H., Ben-David, I., and Wu, S., "Five-Primary-Color LCDs," Journal of Display Technology 6(1), 3-7 (2010).

[4] Yoshiyama, K., Teragawa, M., Yoshida, A., Tomizawa, K., Nakamura, K., Yoshida, Y., and Yamamoto, Y., "Power-saving: A new advantage of multi-primary color displays derived by numerical analysis," in [SID Symposium Digest of Technical Papers], 41, 416-418 (May 2010).

[5] Tsai, C.-C., Lin, F.-C., Huang, Y.-P., , and Shieh, H.-P. D., "RGBW 4-in-1 LEDs for backlight system for ultra-low power consumption field-sequential-color LCDs," in [SID Symposium Digest of Technical Papers], 41, 420-423 (May 2010).

[6] Ueki, S., Nakamura, K., Yuhichi, Yoshida, Mori, T., Tomizawa, K., Narutaki, Y., Itoh, Y., , and Okamoto, K., "Five-Primary-Color 60-inch LCD with novel wide color gamut and wide viewing angle," in [SID Symposium Digest of Technical Papers], 40, 927-930 (June 2009).

[7] Rodríguez-Pardo, C. E., Sharma, G., Feng, X., Speigle, J., and Sezan, I., "Optimal gamut volume design for three primary and multiprimary display systems," in [Proc. SPIE: Color Imaging XVII: Displaying, Hardcopy, Processing, and Applications], Eschbach, R., Marcu, G. G., and Rizzi, A., eds., 8292, 82920C,1-7 (Jan. 2012).

[8] Wen, S., "Design of relative primary luminances for four-primary displays," Displays 26(4-5), 171 - 176 (2005).

[9] Wen, S., "Primary selection for the displays with RGB-LED primaries considering the primary optical power and color gamut," in [SID Symposium Digest of Technical Papers], 39, 787-790 (May 2008).

[10] Roth, S., Ben-David, I., Ben-Chorin, M., Eliav, D., and Ben-David, O., "Wide gamut, high brightness multiple primaries single panel projection displays," in [SID Symposium Digest of Technical Papers], 34, 118-121, Society for Information display (2003).

[11] Ajito, T., Obi, T., Yamaguchi, M., and Ohyama, N., "Expanded color gamut reproduced by six-primary projection display," Proc. SPIE 3954, 130 (2000).

[12] MacAdam, D. L., "Maximum visual efficiency of colored materials," J. Opt. Soc. Am. 25(11), 361-367 (1935).

[13] Hinnen, K. and Langendijk, L., "Design Considerations for Wide Gamut Displays," in [Proc. ISEST/SID Eighteenth Color and Imaging Conference: Color Science and Engineering Systems, Technologies, and Applications], 11-16 (8-12 Nov. 2010).

[14] Wen, S., "A method for selecting display primaries to match a target color gamut," Journal of the Society for Information Display 15, 1015 (2007).

[15] Rodríguez-Pardo, C. E., Sharma, G., Speigle, J., Feng, X., and Sezan, I., "Efficient computation of display gamut volumes in perceptual spaces," in [Proc. ISET/SID Nineteenth Color and Imaging Conference: Color Science and Engineering Systems, Technologies, and Applications], 132-138 (7-11 Nov. 2011).

[16] Masaoka, K., "Fast and accurate model for optimal color computation," Optics letters 35(12), 2031-2033 (2010).

[17] Cheng, Y., Liu, X., and Li, H., "A color temperature adjustment method for multiprimary displays using nonlinear programming," Color Res. Appl. 34(3), 201-204 (2009).

[18] Storn, R. M. and Price, K. V., "Differential evolution-a simple and efficient heuristic for global optimization over continuous spaces," Journal of global optimization 11(4), 341-359 (1997).

[19] Mezura-Montes, E., Velázquez-Reyes, J., and Coello Coello, C., "Modified differential evolution for constrained optimization," in [Evolutionary Computation, 2006. CEC 2006. IEEE Congress on], 25-32, IEEE (2006).

[20] "Quattron technology ${ }^{\mathrm{TM}}$." http://www.aquos-world.com/en/product/4_color_innovation.html. accessed November 2013. 